\title{
Association of Notch3 single-nucleotide polymorphisms and lacunar infarctions in patients
}

\author{
YING LI ${ }^{1,2}$, NAN LIU ${ }^{2}$, HUI $\mathrm{CHEN}^{2}$, YONGHUA HUANG $^{2}$ and WEIWEI ZHANG ${ }^{2}$ \\ ${ }^{1}$ Postgraduate School, Third Military Medical University, Chongqing 400300; \\ ${ }^{2}$ Department of Neurology, Military General Hospital of Beijing PLA, Beijing 100700, P.R. China
}

Received September 25, 2015; Accepted November 25, 2015

DOI: $10.3892 /$ etm.2015.2898

\begin{abstract}
Cerebrovascular disease is a leading cause of morbidity and mortality worldwide, which is influenced by genetic and environmental factors. The aim of the present study was to examine the association between single-nucleotide polymorphisms (SNPs) in Notch3 exons 3-6 and lacunar infarction by comparing SNPs between control subjects and those with lacunar infarction. A single-center case-control study was conducted to investigate the association between Notch3 SNPs and risk of stroke. A total of 140 patients were included in the study, 30 of whom had no infarction (control) and 110 had lacunar infarction. Lacunar patients were divided into the 'pure lacunar' and 'lacunar + leukoarasis' groups based on brain imaging. All the patients were of Chinese Han ethnicity, and the male to female ratio was 84:56. Patient clinical histories included hypertension, diabetes mellitus (DM), hyperlipidemia, and heart disease were recorded. The Notch3 sequence was obtained from the National Centser for Biotechnology Information database. Notch3 was amplified by polymerase chain reaction from whole blood samples, and exons 3-6 were sequenced to identify SNPs. The result showed that there was no significant difference in the prevalence of hypertension, DM, hyperlipidemia, and heart disease between the control and lacunar infarction patients. Notabley, the age of the lacunar + leukoarasis patients was significantly higher than that of the control and pure lacunar patients $(\mathrm{P}<0.05)$. Eight SNPs were detected at low frequencies, and only rs3815388 and rs1043994 exhibited slightly higher frequencies. A $\chi^{2}$ test indicated that Notch 3 SNPs, particularly rs1043994, were associated with lacunar infarction $(\mathrm{P}<0.05)$. In conclusion, the result of the present study have shown that Notch3 SNPs, particularly rs1043994, are associated with lacunar infarction.
\end{abstract}

Correspondence to: Dr Weiwei Zhang, Department of Neurology, Military General Hospital of Beijing PLA, 5 Nanmengcang, Beijing 100700, P.R. China

E-mail: zhangvv2011@126.com

Key words: Notch3 gene, single-nucleotide polymorphisms, lacunar, infarction, cerebrovascular disease, leukoaraiosis

\section{Introduction}

Cerebrovascular disease (CVD) is a leading cause of morbidity and mortality worldwide (1) Hypertension, diabetes mellitus (DM), dyslipidemia, and smoking are risk factors for ischemic CVD (ICVD) (2-4). Atherosclerosis is the most common cause of ICVD (5), but gene mutations are also associated with this disease. CVD may result from genetic and environmental factors (6,7). Sickle cell anemia, Fabry disease, and cerebral autosomal dominant arteriopathy with subcortical infarcts and leukoencephalopathy (CADASIL) are caused by single gene defects associated with ICVD (6-8). In particular, CADASIL is a small-vessel disease that can cause stroke. The four cardinal features of CADASIL are aura, cerebrovascular ischemic events, mood disturbances and dementia. Lacunar ICVD is frequently associated with CADASIL and is found in $\sim 85 \%$ of symptomatic CADASIL patients (9).

Notch 3 mutations have been shown to cause CADASIL (10). Notch 3 has 31 exons, although most of the mutations are located in exons 2-24, which encode the extracellular domain (ECD) of the Notch3 receptor. Mutations in the parts of exons 3 and 4 that code for the first five epidermal growth factor-like repeats (EGFRs) were present in $70 \%$ of the subjects with CADASIL (11). These mutations result in a gain or loss of cysteine residues in one of the 34 EGFRs in the ECD of the Notch3 protein, which are very important in vascular system development and maturity. Numerous polymorphisms have been identified in the coding sequence of Notch3, some of which have led to amino acid substitutions (10). However, whether these polymorphisms affect the Notch3 signaling pathway or are involved in CVD is unknown.

The aim of the present study was to examine the association between single-nucleotide polymorphisms (SNPs) in Notch3 exons 3-6 and lacunar ischemic stroke (LIS). Blood samples were collected from the control subjects and those with LIS to analyze Notch3 exons 3-6 to determine whether Notch3 SNPs are associated with lacunar ICVD.

\section{Patients and methods}

Ethics approval. The study protocol was approved by the Ethics Committee of Beijing Military General Hospital (Beijing, China). All the participants included in the study provided written informed consent. 
Table I. Clinical characteristics of included subjects.

\begin{tabular}{lcccc}
\hline Characteristics & Control & Lacunar & Pure lacunar & Lacunar + leukoaraiosis \\
\hline Male:female & $17: 13$ & $67: 43$ & $53: 27$ & $14: 16$ \\
Age, years (mean \pm SD) & $63.03 \pm 12.25$ & $69.05 \pm 10.89$ & $67.74 \pm 11.04$ & $73.24 \pm 9.43^{\text {a }}$ \\
Hypertension, no. (\%) & $12(40.0)$ & $62(56.4)$ & $42(52.5)$ & $20(66.6)$ \\
DM, no (\%) & $11(36.7)$ & $35(31.8)$ & $23(28.8)$ & $12(40.0)$ \\
Heart disease, no. $(\%)$ & $10(33.3)$ & $28(25.4)$ & $20(25.0)$ & $8(26.6)$ \\
Hyperlipidemia, no. $(\%)$ & $6(20.0)$ & $37(33.6)$ & $28(35.0)^{\mathrm{a}}$ & $9(30.0)$
\end{tabular}

${ }^{\mathrm{a}} \mathrm{P}<0.05$. DM, diabetes mellitus; $\mathrm{SD}$, standard deviation.

Patients. A total of 140 Chinese Han patients, including 110 lacunar patients and 30 controls (>40 years old) admitted to the Department of Neurology, Military General Hospital of Beijing (Beijing, China) between June and December 2010 were included in the study. Demographic factors (gender and age) were recorded. Subjects with LIS were enrolled, as were subjects without infarction (as controls). ICVD was confirmed by magnetic resonance imaging (MRI) or computed tomography $(\mathrm{CT})$ brain imaging.

Lacunar infarcts were defined as parenchymal defects with a signal intensity corresponding to that of the cerebrospinal fluid in all the sequences and were $\leq 15 \mathrm{~mm}$ in diameter, as determined by brain imaging (MRI or CT). Leukoaraiosis was defined as changes in white matter diffusion. These changes were observed on CT scans as bilateral patchy or diffuse areas of hypodensity with ill-defined margins or hyperintensities on T2-weighted MRI involving the periventricular and centrum semiovale white matter.

Clinical history. Clinical history of the participants included CVD, myocardial infarction, atrial fibrillation, hypertension, DM, and hyperlipidemia [hypercholesterolemia; high levels of triglycerides (TGs) and low-density lipoprotein cholesterol (LDL-C)]. Hypertension was defined as a mean blood pressure of $>140 / 90 \mathrm{mmHg}$ or the use of an antihypertensive agent. DM was defined as a fasting glucose level of $\geq 6.1 \mathrm{mmol} / 1$, random non-fasting glucose level of $\geq 11.1 \mathrm{mmol} / 1$, or the use of anti-diabetic medication. Hypercholesterolemia was defined as a total serum cholesterol level of $>5.6 \mathrm{mmol} / \mathrm{l}$. High levels of TGs and LDL-C were defined as total serum TG >1.7 mmol/1 and total serum LDL-C $>2.7 \mathrm{mmol} / \mathrm{l}$, respectively.

Brain imaging. MRI was conducted using a 3.0-T system (Discovery MR750; GE Healthcare, Waukesha, WI, USA). The brain imaging protocol (slice thickness, $5 \mathrm{~mm}$; interslice thickness, $1.5 \mathrm{~mm}$ ) employed the following parameters: T1 fluid-attenuated inversion recovery images (TR, 1,750 msec; TE, $23 \mathrm{msec}$; TI, $780 \mathrm{msec}$ FOV, $24 \mathrm{~cm}$ ) and T2-weighted images (TR, 7,498 msec; TE, $105 \mathrm{msec}$; FOV, $24 \mathrm{~cm}$ ). CT (GE LightSpeed VCT 64 system; GE Healthcare, Waukesha, WI, USA) was conducted at a slice thickness of $9 \mathrm{~mm}$.

Gene analyses. To identify Notch3 SNPs, genomic DNA was obtained from blood using a Human Blood DNA kit (Qiagen, Hilden, Germany). DNA was stored at $-20^{\circ} \mathrm{C}$ prior to genotyping. The region of interest was amplified by polymerase chain reaction (PCR) to analyze the SNPs in Notch3 exons 3-6.

The primers for exons 3 and 4 were: forward: 5'-GTT TGCTGCTCTGTTTCCCTG-3' and reverse: 5'-GGCACA GTCGTAAGTGAGGT-3'. PCR conditions consisted of one cycle of $10 \mathrm{~min}$ at $95^{\circ} \mathrm{C}, 36$ cycles of $30 \mathrm{sec}$ at $94^{\circ} \mathrm{C}$ and $1 \mathrm{~min}$ at $65^{\circ} \mathrm{C}$, followed by $30 \mathrm{~min}$ at $72^{\circ} \mathrm{C}$ in a GeneAmp PCR system 2400 (PerkinElmer, Inc., Foster City, CA, USA). The PCR product was 656 base pairs. For the reverse reaction of exons 5 and 6, the primers used were: forward: 5'-AGAAAA CGGCCACTCACCAG-3' and reverse 5'-ACACCGATGTCT CAATGGGG-3', at an annealing temperature of $55^{\circ} \mathrm{C}$. The PCR product was 415 base pairs.

Direct sequencing of the PCR product was performed by a genomic company in Chongqing, China (Genemine Biotechnology Co., Ltd., Chongqing, China), and GeneTools software (Gene Tools, LLC, Philomath, OR, USA) was used to identify the SNPs. The position of the nucleotide sequence was based on the reference sequence obtained from the National Center for Biotechnology Information (NCBI) nucleotide database. The SNP database of the NCBI database was used (www.ncbi.nlm.nih.gov/SNP/).

Statistical analysis. Data were presented as mean \pm SD. The differences in the genotype frequencies and other risk factors were analyzed by the $\chi^{2}$ test. Mean ages in the two groups and allele frequencies were compared using the independent samples t-test. The $\chi^{2}$ tests were used to determine the relationship between the SNPs and LIS. Statistical analyses were conducted using SPSS v16.0 (SPSS, Inc., Chicago, IL, USA). $\mathrm{P}<0.05$ was considered to indicate statistically significant results.

\section{Results}

Patient demographics and disease characteristics. A total of 110 LIS (67 males) and 30 control (17 males) patients participated in the study. LIS patients were sub-classified as 'pure lacunar' or 'lacunar + leukoaraiosis'. The age of the subjects in the lacunar + leukoaraiosis group $(73.24 \pm 9.43$ years) was significantly greater than that of the control $(63.03 \pm 12.25$ years $)$ and pure lacunar $(67.74 \pm 11.04$ years $)$ groups $(\mathrm{P}<0.05)$. The characteristics of the subjects are summarized in Table I.

Approximately $25.4 \%(n=28), 56.4 \%(n=62)$, and $31.8 \%(n=35)$ of subjects with LIS also had heart disease, hypertension, 


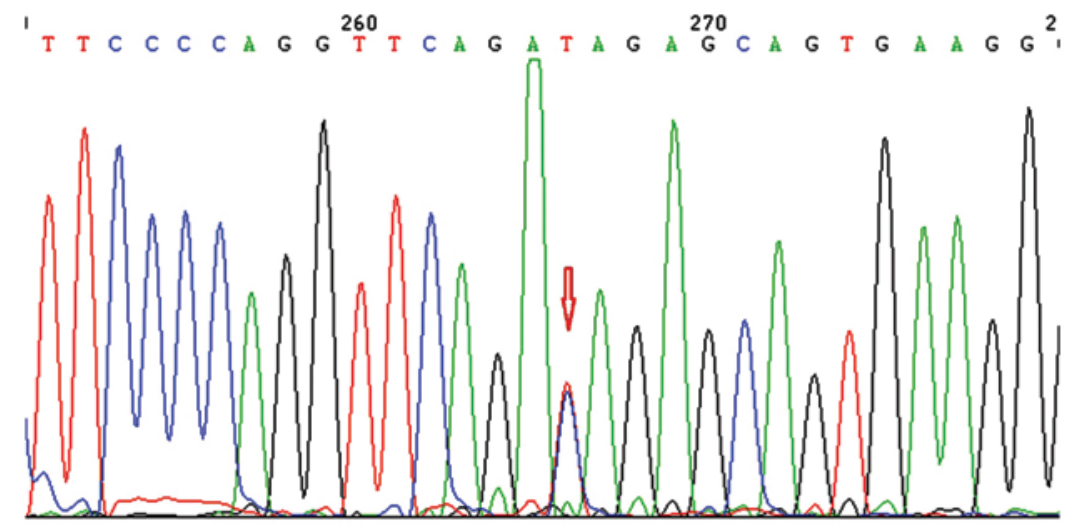

Figure 1. The rs3815188 CT (red arrow) single-nucleotide polymorphism.

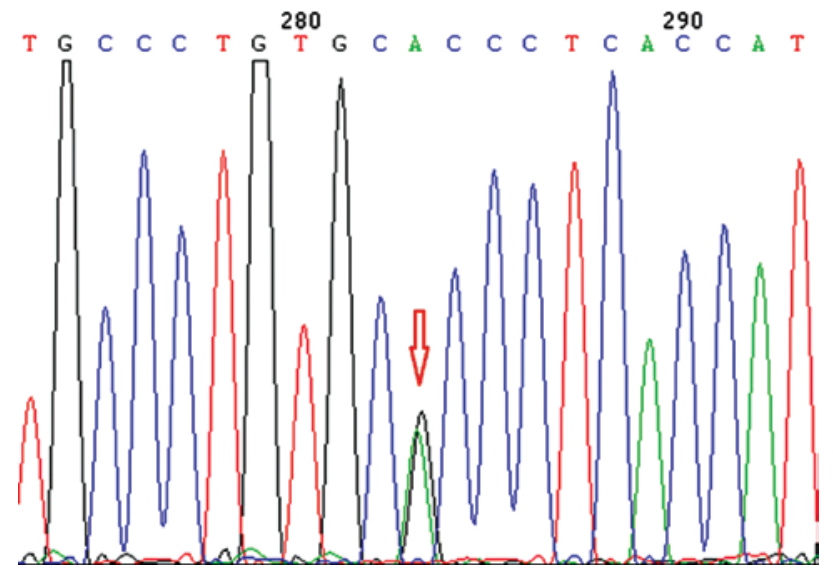

Figure 2. The rs1043994 AG (red arrow) single-nucleotide polymorphism.

and DM, respectively, whereas $33.3 \%(n=10), 40.0 \%(n=12)$, and $36.7 \%(n=11)$ of the control group had these risk factors, respectively. Significant differences were not observed in the two groups with regard to clinical history. Hyperlipidemia was observed in $33.6 \%(n=37)$ of subjects with lacunar ICVD and $20.0 \%(n=6)$ of individuals in the control group. This difference was not significant, although the rate of hyperlipidemia in subjects with LIS $(35.0 \%, \mathrm{n}=28)$ was significantly higher than that in the control group $(20.0 \%, \mathrm{n}=6)(\mathrm{P}<0.05)$.

Notch3 SNPs and LIS. A total of 37 SNPs were identified in Notch3 exons 3-6. Of those SNPs, only rs146810942, rs135069047, rs3815188, rs202157633, rs142778401, rs1043994, rs2285981 and rs149307620 were present in the current study. Of these 8 SNPS, only rs3815188 and rs1043994 were identified at higher frequencies. Thus, we only analyzed the association between LIS and the rs3815188 (Fig. 1) and rs1043994 (Fig. 2) SNPs. rs3815188 and rs1043994 were identified in exons 3 and 4 , respectively (Table II).

The frequency of the TT genotype in rs3815188 was higher in the LIS group than in the control group, albeit this result was not statistically significant $(\mathrm{P}>0.05)$. However, we did not observe any association between the allelic frequency of rs3815188 and LIS. The frequency of the AG genotype in rs1043994 was higher in the lacunar + leukoaraiosis group than in the control group $(\mathrm{P}<0.05)$. The $\chi^{2}$ test revealed an association between rs1043994 allelic frequency and LIS.
SNP characteristics and clinical history. The association between rs3815188 and rs1043994 allelic frequencies and hypertension, DM, hyperlipidemia, and heart disease were assessed, albeit no significant difference was observed (Table III).

We also found a new SNP located at chromosome position 15302941(C588T) that was not included in the NCBI SNP database.

\section{Discussion}

CADASIL is a hereditary micro-angiopathic condition that can cause stroke in young adults. It is a non-atherosclerotic, non-amyloid angiopathy that affects the small penetrating arteries in the white matter (12). The mean age at onset is approximately 45 years (range, 30-70 years), and most ischemic stroke patients with CADASIL present with lacunar syndromes (pure motor stroke, pure sensory stroke, sensorimotor paralysis, ataxic hemiparesis, or dysarthria) (13). Notch 3 mutations are the main cause of CADASIL. Notch3 is located on chromosome 19q13.1-13.2, and the Notch3 receptor protein is a single transmembrane protein (14) with intracellular and intercellular domains. Most mutations result in a gain or loss of cysteine residues in one of the 34 EGFRs in the ECD of the Notch3 protein. These changes subsequently affect small vessel structural integrity (15).

A number of SNPs were identified in the coding sequence of Notch3, some of which led to amino acid substitutions. In 
Table II. Genotype distributions.

\begin{tabular}{lcccc}
\hline Genotype & Control, no. $(\%)$ & Lacunar, no. $(\%)$ & Pure lacunar, no. $(\%)$ & Lacunar + leukoaraiosis, no. $(\%)$ \\
\hline rs3815188 & & & & $13(43.3)$ \\
CC & $15(50)$ & $47(42.7)$ & $34(42.5)$ & $6(20.0)$ \\
TT & $1(3.3)$ & $22(20.0)$ & $16(20.0)$ & $11(36.7)$ \\
CT & $14(46.7)$ & $41(37.3)$ & $30(37.5)$ & \\
rs1043994a & & & & $14(46.7)$ \\
AA & $21(70.0)$ & $5(4.5)$ & $62(77.5)$ & $16(53.3)^{\mathrm{b}}$ \\
GG & $9(30.0)$ & $76(69.1)$ & $13(16.3)$ & \\
AG & $29(26.4)$ & & \\
\hline
\end{tabular}

${ }^{\mathrm{a}} \chi^{2}$ test, $\mathrm{P}<0.05 ;{ }^{\mathrm{b}} \mathrm{P}<0.05$.

Table III. SNP characteristics and clinical history.

\begin{tabular}{|c|c|c|c|c|c|c|}
\hline \multirow[b]{2}{*}{ Characteristics } & \multicolumn{3}{|c|}{ rs3815388, no. (\%) } & \multicolumn{3}{|c|}{ rs 1043994, no. (\%) } \\
\hline & $\mathrm{CC}$ & $\mathrm{TT}$ & $\mathrm{CT}$ & AA & GG & $\mathrm{AG}$ \\
\hline Hypertension & $33(44.6)$ & $13(17.6)$ & $28(37.8)$ & $1(1.4)$ & $55(74.3)$ & $18(24.3)$ \\
\hline DM & $18(39.1)$ & $7(15.2)$ & $21(45.7)$ & 0 & $34(73.9)$ & $12(26.1)$ \\
\hline Hyperlipidemia & $21(48.8)$ & $10(23.3)$ & $12(27.9)$ & $2(4.6)$ & $31(72.1)$ & $10(23.3)$ \\
\hline Heart disease & $15(39.5)$ & $4(10.5)$ & $19(50.0)$ & $2(5.2)$ & $27(71.1)$ & $9(23.7)$ \\
\hline
\end{tabular}

SNP, single-nucleotide polymorphism; DM, diabetes mellitus.

Notch3 exons 3-6, there are 37 SNPs, the majority of which do not lead to amino acid substitutions. However, whether these SNPs affect the vessel wall remains to be determined. We found that 8 SNPs were present in our patients, and for most of these, the frequency was low. Of these, only rs3815188 and rs1043994 had higher frequencies, although they did not cause amino acid substitution. In lacunar patients, rs3815188TT frequency was higher than that in the pure lacunar and lacunar + leukoaraiosis groups. Additionally, there was no significant difference in the rs3815188 frequency when compared to the control, as determined by the $\chi^{2}$ test. There were significant differences between the control and lacunar patients with respect to the rs1043994 frequency. However, in the lacunar + leukroarasis patients, rs1043994AG had a frequency higher than that in the control subjects. The $\chi^{2}$ test revealed that rs1043994 was significantly associated with LIS.

Previous findings $(16,17)$ showed that some Notch3 SNPs had no association with CVD. However, these studies only analyzed $1 \mathrm{SNP}, \mathrm{C} 381 \mathrm{~T}$, and the type of stroke was not identified. However, in another study (18) all of the common Notch3 SNPs $(n=888)$, and 4 of these (rs1043994, rs10404382, rs10423702 and rs1043997) were screened and found to be significantly associated with the presence and progression of white matter lesions. Our results are consistent with that study, showing that Notch3 mutations and SNPs are important for the integrity and function of small vessels. Additionally, findings of a large case-control study of Chinese patients showed that individuals with a combination of MTHFR 677TT, ALOX5AP 2354AA, and NOTCH3 381TT/TC had a significantly higher risk of thrombotic stroke (19) $(\mathrm{OR}=3.165,95 \%$ confidence interval: 1.461-6.858; $\mathrm{P}=0.003)$.

Moreover, in a study on CADASIL from Chinese patients it was identified that the mutational spectrum and primary clinical characteristics of patients with CADASIL were similar to those in Caucasians $(20,21)$. However, migraine with aura and abnormal white matter in the temporal pole were less common than in Caucasians, while brainstem involvement was more common. That study revealed that Notch3 mutations in Chinese patients had different effects on vessels.

Our findings have shown that, although the 2 SNPs on Notch3 exons 3-6 did not result in amino acid substitutions, the effect of these 2 SNPs on vascular development remains to be determined, although rs1043994 was associated with LIS. The Notch3 receptor participates in vessel development and maturation (22). Notch receptor signaling is involved in the control of proliferation of smooth muscle cells (SMCs) and maintenance of undifferentiated SMCs. In arteries, Notch3 protein is the predominant receptor in vascular SMCs, and signaling is initiated following binding to its ligand, Jagged1 (23). Notch3 gene mutation results in arteriopathy and leukoencephalopathy (24). However, how ligand-presenting strategies affect Notch signaling and subsequent upregulation of SMC differentiation remain to be elucidated. If Notch 3 abnormalities are detected earlier, it may be possible to prevent CVD.

No differences were identified with regard to Notch3 SNPs between the pure lacunar and lacunar + leukoaraiosis 
groups. The prevalence of hypertension was greater in the lacunar + leukoaraiosis group than that in the control and pure lacunar groups, suggesting that hypertension is an important factor in small-vessel disease. The mean age of the lacunar + leukoaraiosis group was higher than that of the pure lacunar and control groups $(\mathrm{P}<0.05)$. Thus, age may be another risk factor for leukoaraiosis. rs1043994AG had a higher frequency in the lacunar + leukoaraiosis group than that in the control and pure lacunar groups, suggesting that this gene type is important for small vessels.

The primary limitation of this study was the low number of participants (especially in the control group). Additional studies conducted in larger populations are required to confirm our findings. Additionally, we did not study SNPs from whole Notch 3 sequences, which is imperative to elucidate the association between the Notch3 SNPs and lacunar infarctions. The mechanism by which Notch3 SNPs affects small vessels should also be investigated.

In conclusion, the results of the present study suggest that Notch3 SNPs are likely to be associated with lacunar infarction. In particular, we found that rs1043994 was associated with lacunar infarctions.

\section{References}

1. Wang YJ, Zhang SM, Zhang L, Wang CX, Dong Q, Gao S, Huang RX, Huang YN, Lv CZ, Liu M, et al: Chinese guidelines for the secondary prevention of ischemic stroke and transient ischemic attack 2010. CNS Neurosci Ther 18: 93-101, 2012.

2. Bartsch JA, Teare GF, Neufeld A, Hudema N and Muhajarine N: Secondary prevention of stroke in Saskatchewan, Canada: ๆypertension control. Int J Stroke 8: 32-38, 2013.

3. Hankey GJ, Wong KS, Chankrachang S, Chen C, Crimmins D, Frayne J, Kim JS, Li Y, Liou CW, Merican JS, et al; Working Group on Stroke and Lipids Management in Asia Consensus Panel: Management of cholesterol to reduce the burden of stroke in Asia: consensus statement. Int J Stroke 5: 209-216, 2010.

4. Allen CL and Bayraktutan U: Risk factors for ischaemic stroke. Int J Stroke 3: 105-116, 2008.

5. Kim JS, Nah HW, Park SM, Kim SK, Cho KH, Lee J, Lee YS, Kim J, Ha SW, Kim EG, et al: Risk factors and stroke mechanisms in atherosclerotic stroke: Intracranial compared with extracranial and anterior compared with posterior circulation disease. Stroke 43: 3313-3318, 2012

6. Meschia JF, Worrall BB and Rich SS: Genetic susceptibility to ischemic stroke. Nat Rev Neurol 7: 369-378, 2011.

7. Zhu Y, Wang J, Wu Y, Wang G and Hu B: Two novel mutations in NOTCH3 gene causes cerebral autosomal dominant arteriopathy with subcritical infarct and leucoencephalopathy in two Chinese families. Int J Clin Exp Pathol 8: 1321-1327, 2015.

8. Bevan S and Markus HS. Genetics of common polygenic ischaemic stroke: current understanding and future challenges. Stroke Res Treat 2011: 179061, 2011.

9. Viswanathan A, Gschwendtner A, Guichard JP, Buffon F, Cumurciuc R, O'Sullivan M, Holtmannspötter M, Pachai C, Bousser MG, Dichgans M, et al: Lacunar lesions are independently associated with disability and cognitive impairment in CADASIL. Neurology 69: 172-179, 2007.
10. Pescini F, Nannucci S, Bertaccini B, et al: The cerebral autosomal-dominant arteriopathy with subcortical infarcts and leukoencephalopathy (CADASIL) scale. Stroke 43: 2871-2876, 2012.

11. Dichgans M, Ludwig H, Müller-Höcker J, Messerschmidt A and Gasser T: Small in-frame deletions and missense mutations in CADASIL: 3D models predict misfolding of Notch3 EGF-like repeat domains. Eur J Hum Genet 8: 280-285, 2000.

12. Okeda R, Arima K and Kawai M: Arterial changes in cerebral autosomal dominan arteriopathy with subcortical infarcts and leukoencephalopathy (CADASIL) in relation to pathogenesis of diffuse myelin loss of cerebral white matter. Stroke 33: 2565-2569, 2002

13. Tang SC, Jeng JS, Lee MJ and Yip PK: Notch signaling and CADASIL. Acta Neurol Taiwan 18: 81-90, 2009.

14. Joutel A, Monet M, Domenga V, Riant F and Tournier-Lasserve E: Pathogenic mutations associated with cerebral autosomal dominant arteriopathy with subcortical infarcts and leukoencephalopathy differently affect Jagged1 binding and Notch3 activity via the RBP/JK signaling Pathway. Am J Hum Genet 74: 338-347, 2004.

15. Domenga V, Fardoux P, Lacombe P, Monet M, Maciazek J, Krebs LT, Klonjkowski B, Berrou E, Mericskay M, Li Z, et al: Notch 3 is required for arterial identity and maturation of vascular smooth muscle cells. Genes Dev 18: 2730-2735, 2004.

16. Ito D, Tanahashi N, Murata M, Sato H, Saito I, Watanabe K and Fukuuchi Y: Notch3 gene polymorphism and ischaemic cerebrovascular disease. J Neurol Neurosurg Psychiatry 72: 382-384, 2002.

17. Mizuno T, Makino M, Fujiwara Y and Nakajima K: Lack of association between NOTCH3 gene polymorphism and cerebrovascular disease in Japanese patients. Ann N Y Acad Sci 977: 252-257, 2002

18. Schmidt H, Zeginigg M, Wiltgen M, Freudenberger P, Petrovic K, Cavalieri M, Gider P, Enzinger C, Fornage M, Debette S, et al; CHARGE consortium Neurology working group: Genetic variants of the NOTCH3 gene in the elderly and magnetic resonance imaging correlates of age-related cerebral small vessel disease. Brain 134: 3384-3397, 2011

19. Liu J, Sun K, Bai Y, Zhang W, Wang X, Wang Y, Wang H, Chen J, Song $\mathrm{X}$, Xin Y, et al: Association of three-gene interaction among MTHFR, ALOX5AP and NOTCH3 with thrombotic stroke: a multicenter case-control study. Hum Genet 125: 649-656, 2009.

20. Wang Z, Yuan Y, Zhang W, Lv H, Hong D, Chen B, Liu Y, Luan $\mathrm{X}$, Xie S and Wu S: NOTCH3 mutations and clinical features in 33 mainland Chinese families with CADASIL. J Neurol Neurosurg Psychiatry 82: 534-539, 2011.

21. Liao YC, Hsiao CT, Fuh JL, et al: Characteriazation of CADASIL among the Han Chinese in Taiwan: Distinct Genotypic and Phenotypic Profiles. PLoS One 10: e0136501, 2015.

22. Boulos N, Helle F, Dussaule JC, Placier S, Milliez P, Djudjaj S, Guerrot D, Joutel A, Ronco P, Boffa JJ, et al: Notch3 is essential for regulation of the renal vascular tone. Hypertension 57: 1176-1182, 2011.

23. Caolo V, Schulten HM, Zhuang ZW, Murakami M, Wagenaar A, Verbruggen S, Molin DG and Post MJ: Soluble Jagged-1 inhibits neointima formation by attenuating Notch-Herp2 signaling. Arterioscler Thromb Vasc Biol 31: 1059-1065, 2011.

24. Pippucci T, Maresca A, Magini P, Cenacchi G, Donadio V, Palombo F, Papa V, Incensi A, Gasparre G, Valentino ML, et al: Homozygous NOTCH3 null mutation and impaired NOTCH3 signaling in recessive early-onset arteriopathy and cavitating leukoencephalopathy. EMBO Mol Med 7: 848-858, 2015. 